\title{
Modification of energy and angular spectra of binary-encounter emission in fast ion-atom collisions assisted by a low-frequency electromagnetic field
}

\author{
A. B. Voitkiv ${ }^{1,2}$ and J.Ullrich ${ }^{1,2}$ \\ Physics Department, University of Freiburg, Hermann-Herder-Strasse 3, D-79104 Freiburg, \\ Germany \\ Max-Planck-Institüt für Kernphysik, Saupfercheckweg 1, D-69117 Heidelberg, Germany
}

(J.Phys.B, accepted)

\begin{abstract}
We consider the influence of a weak low-frequency electromagnetic field on the binary-encounter electron emission in fast ion-atom collisions. It is shown that the electromagnetic field, which would have no impact on the collisionfree system, can be very effectively coupled to the projectile-target system in collisions where an electron experiences strong acceleration during the process of emission. In such a case a large amount of energy is exchanged between the system and the field which can result in profound modifications of the energy and angular distributions of the emitted electron. It is also found that at a certain collision geometry a large energy exchange with the field has only very weak impact on the electron spectra. This might suggest that, in field-assisted ion-atom collisions, a heavy particle, whose direct interaction with electromagnetic field is negligible, can be rather effectively coupled to the field in an indirect way by 'using' an electron as a mediator.
\end{abstract}

PACS:34.10.+x, 34.50.-s, 34.50.Fa, 34.50.Rk 


\section{INTRODUCTION}

One of the prominent features in electron emission in fast ion-atom collisions is represented by the so called binary-encounter emission (see e.g. [1], where this emission is discussed in details, for a general review of the field of fast ion-atom collisions see [1]- [4], where also references to original works can be found). This emission occurs in such collision events, where the interaction of the electron with the residual atomic target can be neglected during the collision and, thus, the ionization process is determined by two-body kinematics. In the binary-encounter emission the electron velocity $v_{e}$ in the final state is given by $v_{e} \approx 2 v_{p} \cos \vartheta_{k}$, where $v_{p}$ is the projectile-ion velocity and $\vartheta_{k}<\pi / 2$ is the electron emission angle with respect to the projectile velocity $\mathbf{v}_{p}$. For the binary emission in fast collisions, where the projectile velocity $v_{p}$ is much higher than a typical orbiting velocity $v_{0}$ of the active electron in the target initial state, the change in the electron velocity can be quite large. Since the effective collision time, where this change occurs, is rather short, it means that electrons, which are emitted at angles $\vartheta$ not close to $\pi / 2$, experience a very strong acceleration.

The investigations of the influence of a laser field on elementary scattering (collision) processes are of interest because of two main reasons. One reason is that the processes can be important in applied areas, such as plasma heating or laser-driven fusion. The other reason is that the field-assisted processes are quite interesting per se, especially from the point of view of fundamental collision physics. Inclusion of electromagnetic fields in atomic collisions introduces new degrees of freedom and can substantially affect the collision dynamics. This is expected to be especially the case in collisions where charged particles are strongly accelerated. As is known from electrodynamics, very effective coupling and energy exchange between a charged particle and radiation field occur if the particle is strongly accelerated. This property clearly manifests itself in the field-assisted electron-atom scattering (for a recent review of this topic see [5], where also a large number of references to original articles can be found). For example, in the simplest case of the field-assisted electron-target scattering, represented by electron potential scattering ${ }^{1}$, the energy exchanged with the field and, thus, the field effect are proportional to the change in the electron velocity.

The field-assisted binary-encounter emission in fast ion-atom collisions was considered in [6]. In that paper the cross section was derived for the binary-encounter electron emission in the presence of a weak low-frequency electromagnetic field of linear polarization and an example of energy spectra of the emitted electron was briefly discussed. This subject, however, seems to be interesting enough to deserve a more detailed analysis. In the present paper we continue to study the modifications in the binary-encounter emission in fast ionatom collisions in the presence of a laser field. We will give a different derivation for the cross section of the electron emission in field-assisted collisions. This derivation procedure is simpler, compared to that formulated in [6], and it can be applied for the case of an arbitrary (elliptic) polarization. We shall study more in detail the energy spectra of emitted electrons and consider their angular distributions. In the projectile frame the field-assisted emission can be regarded as 'bound-free' electron scattering on the ion where the electron,

\footnotetext{
${ }^{1}$ Below this process will also be termed as free-free transitions or free-free scattering.
} 
initially bound in a moving atom, makes a transition into a continuum state. We will discuss the similarity and differences in the free-free and 'bound-free' electron scattering. We shall also briefly touch upon the field-assisted binary emission in collisions with a neutral atomic projectile and the possibility to observe the predicted effects experimentally.

Atomic units are used throughout except where otherwise stated.

\section{GENERAL CONSIDERATION}

We shall consider fast nonrelativistic collisions, where $v_{0} \ll v_{p} \ll c$. Here $v_{p}$ is the projectile velocity, $v_{0} \sim Z_{t}$ is a typical orbiting velocity of an electron in the target ground state, $Z_{t} \sim 1$ is the nuclear charge of a single-electron target and $c=137$ is the speed of light. The target is initially in the ground state. The projectile is a bare nucleus with a charge $Z_{p} \ll v_{p}$. The collision occurs in the presence of a monochromatic electromagnetic field. We shall consider linearly, circularly and elliptically polarized fields with the main emphasis on the cases of linear and circular polarizations. To ensure that a projectile collides with a target when the latter is still in its ground state, the electromagnetic field is supposed to have a weak effect on a collision-free target atom in the ground state. An electromagnetic field will weakly influence a free target atom if the following conditions are fulfilled: i) $F_{0 j} \ll F_{a t}$, where $F_{0 j}(\mathrm{j}=1,2)$ are the amplitudes of the electric component of the electromagnetic field

and $F_{a t} \sim \frac{Z_{t}}{a_{0}^{2}}=Z_{t}^{3}$ a.u. is the typical atomic field in the target ground state; ii) the field frequency $\omega_{0}$ is small compared to the minimum excitation energy of the electron bound in the ground state of the target; iii) there are no multiphoton resonances between the ground and excited states of the target in the presence of the electromagnetic field. It will be seen below that there exists a rather wide range of field intensities whose effect on the ground state of a free target is very weak whereas the field modulation of high-energy continuum states of the electron and the field impact on the collision process can be very substantial.

It is convenient to consider the ion-atom collision in the reference frame where an atom is initially at rest. In this frame the recoil of an atomic nucleus is small and its neglect does not influence the electron emission spectra. Therefore in our consideration we assume the target nucleus to be at rest and take it as the origin.

It is well known that, if the condition $v_{p} \gg Z_{p}$ is fulfilled, many phenomena occurring in high-velocity atomic collisions can be described within the first order Born approximation in the projectile-target interaction. In particular, the first order represents an excellent approximation to describe the field-free binary-encounter emission when the conditions $Z_{p} \ll$ $v_{p}$ and $Z_{p} \ll\left|\mathbf{k}-\mathbf{v}_{p}\right|$ are fulfilled, where $\mathbf{k}$ is the electron momentum in the final state with respect to the target nucleus. The first order approximation seems to be valid also for field-assisted collisions provided the electron velocity in the final state with respect to the projectile is much higher than a typical velocity of the oscillations of a free electron in the laser field.

Within the first order Born approximation the $S$-matrix element for an inelastic collision is given by

$$
S_{f i}=-i \int_{-\infty}^{+\infty} d t \int d^{3} \mathbf{R} \int d^{3} \mathbf{r} \Psi_{f}^{*}(\mathbf{R}, \mathbf{r}, t) W_{i n t}(\mathbf{R}, \mathbf{r}) \Psi_{i}(\mathbf{R}, \mathbf{r}, t)
$$


In Eq.(1) $\Psi_{i}(t)$ and $\Psi_{f}(t)$ are the initial and final states of the collision system which also account for the interaction with a laser field. Further, $W_{i n t}(t)=-\frac{Z_{p}}{|\mathbf{R}-\mathbf{r}|}$ is the Coulomb interaction between the projectile and electron ${ }^{2}, \mathbf{r}$ and $\mathbf{R}$ are the coordinates of the electron and projectile given with respect to the target nucleus.

Neglecting the interaction of the field of a laser with the heavy particles, the states $\Psi_{i}(t)$ and $\Psi_{f}(t)$ are written as

$$
\begin{aligned}
\Psi_{i}(t) & =\frac{1}{\sqrt{V}} \exp \left(i\left(\mathbf{P}_{i} \cdot \mathbf{R}-E_{i} t\right)\right) \psi_{i}(\mathbf{r}, t) \\
\Psi_{f}(t) & =\frac{1}{\sqrt{V}} \exp \left(i\left(\mathbf{P}_{f} \cdot \mathbf{R}-E_{f} t\right)\right) \psi_{f}(\mathbf{r}, t) .
\end{aligned}
$$

Here $\mathbf{P}_{i, f}$ and $E_{i, f}$ are the initial and final momentum and energy of the projectile, respectively, and $V$ is the normalization volume for the projectile. $\psi_{i, f}$ represent the initial and final states of the electron which moves in the field of the target nucleus and is simultaneously embedded in the laser field.

With the help of the identity

$$
\frac{1}{x}=\frac{1}{2 \pi^{2}} \int d^{3} \mathbf{q} \frac{\exp (-i \mathbf{q} \cdot \mathbf{x})}{q^{2}}
$$

the transition matrix element (1) by means of straightforward manipulations can be transformed into

$$
S_{f i}=\frac{i Z_{p}}{2 \pi^{2} V \mathbf{q}^{2}} \int_{-\infty}^{+\infty} d t \int d^{3} \mathbf{r} \psi_{f}^{*}(\mathbf{r}, t) \exp (i \mathbf{q} \cdot \mathbf{r}) \psi_{i}(\mathbf{r}, t)
$$

In the above equation $\mathbf{q}=\mathbf{P}_{i}-\mathbf{P}_{f}$ is the change in the projectile momentum due to the collision.

The initial and final electron states obey the following Schrödinger equation

$$
i \frac{\partial}{\partial t} \psi_{i, f}(t)=\left(H_{a t}+H_{i n t}\right) \psi_{i, f}(t)
$$

where $H_{a t}$ is the Hamiltonian of the free target and $H_{\text {int }}$ is the interaction of the electron with the electromagnetic field. In the velocity gauge and within the dipole approximation the interaction reads

$$
H_{i n t}=\frac{1}{c} \mathbf{A}(t) \cdot \hat{\mathbf{p}}+\frac{A^{2}(t)}{2 c^{2}} .
$$

Here $\mathbf{A}(t)$ is the coordinate-independent vector potential of the electromagnetic field, which is treated as a classical field, and $\hat{\mathbf{p}}$ is the electron momentum operator.

\footnotetext{
${ }^{2}$ Since below we use approximate expressions for the initial and final electron states, dressed by the electromagnetic field, the interaction of the projectile with the target nucleus could formally also contribute to the transition matrix element (1). For the process under consideration, however, this contribution turns out to be negligible.
} 
We have already chosen the field parameters in such a way that the field only very weakly affects the atomic ground state. One should, however, take some care in writing down a wavefunction for the ground state in the velocity gauge. Following [6], the initial electron state will be taken in this gauge as

$$
\psi_{i}(t)=\varphi_{0}(\mathbf{r}) \exp \left(-i \varepsilon_{0} t\right) \exp \left(-i \frac{1}{c} \mathbf{A}(t) \cdot \mathbf{r}\right),
$$

where $\varphi_{0}$ and $\varepsilon_{0}$ are the wavefunction and energy of the ground state of the free target. One can note that the first two terms on the right hand side of (7) represent the initial electron state in the length gauge obtained to zeroth order in $F_{0} / F_{a t}$, i.e. these terms describe the unperturbed atomic ground state. The term $\exp \left(-i \frac{1}{c} \mathbf{A}(t) \mathbf{r}\right)$ is the gauge factor which transforms the initial electron state to the velocity gauge.

Taking into account that $v_{p} \gg Z_{t}, Z_{p}$ and assuming that in the final state the velocities of the electron with respect to both the residual target and projectile are much higher than the typical oscillation velocity of a free electron in the laser field, the final electron state can be well approximated by the so called Coulomb-Volkov ansatz which in the velocity gauge reads

$$
\psi_{f}(t)=\varphi_{\mathbf{k}}(\mathbf{r}) \exp \left(-i \varepsilon_{k} t\right) \exp \left(-i \frac{\mathbf{k}}{c} \cdot \int_{+\infty}^{t} d t^{\prime} \mathbf{A}\left(t^{\prime}\right)-\frac{i}{2 c^{2}} \int_{+\infty}^{t} d t^{\prime} A^{2}\left(t^{\prime}\right)\right) .
$$

Here $\varphi_{\mathbf{k}}(\mathbf{r})$ is the Coulomb continuum state of the electron which moves in the field of the target nucleus and $\varepsilon_{k}=\frac{k^{2}}{2}$ is the final kinetic energy of the emitted electron. Now the situation with the relative importance of Coulomb and electromagnetic fields is reversed compared to that in the initial state. The Coulomb field can exert only a weak influence on the behaviour of a high-energy electron. However, the high-energy continuum states can be strongly influenced by a laser field. This influence is described nonperturbatively by the exponential field-dependent factors in (8) which are inherent to the exact solution for the problem of a free electron in the field of a plane electromagnetic wave [7].

Below we consider separately collisions assisted by fields with linear, circular and elliptic polarization.

\section{A. Linear polarization}

In case of linear polarization the vector potential can be taken as $\mathbf{A}(t)=\mathbf{A}_{0} \cos \omega_{0} t$, where $\mathbf{A}_{0}=\frac{c \mathbf{F}_{0}}{\omega_{0}}, \mathbf{F}_{0}$ is the amplitude of the field strength and $\omega_{0}$ is the field frequency. Using standard expansions, the field-dependent exponentials can be rewritten as

$$
\begin{aligned}
& \exp \left(-\frac{i}{c} \mathbf{A}(t) \cdot \mathbf{r}\right) \exp \left(i \frac{\mathbf{k}}{c} \int^{t} d t^{\prime} \mathbf{A}\left(t^{\prime}\right)\right) \exp \left(\frac{i}{2 c^{2}} \int^{t} d t^{\prime} A^{2}\left(t^{\prime}\right)\right) \\
& =\exp \left(i \frac{F_{0}^{2}}{4 \omega_{0}^{2}} t\right) \sum_{n_{1}, n_{2}, n_{3}=-\infty}^{\infty} J_{n_{1}}\left(\alpha_{l}\right) J_{n_{2}}\left(-\beta_{l}\right) J_{n_{3}}\left(\gamma_{l}\right) \\
& \exp \left(i n_{1} \omega_{0} t+i n_{2}\left(\omega_{0} t+\pi / 2\right)+2 i n_{3} \omega_{0} t\right)
\end{aligned}
$$

where $\alpha_{l}=\frac{\mathbf{F}_{0} \cdot \mathbf{k}}{\omega_{0}^{2}}, \beta_{l}=\frac{\mathbf{F}_{0} \cdot \mathbf{r}}{\omega_{0}}, \gamma_{l}=\frac{F_{0}^{2}}{8 \omega_{0}^{3}}$ and $J_{n}(x)$ is the Bessel function. Using the Graf's theorem for the Bessel functions (see e.g. [8]) the double sum over $n_{1}$ and $n_{2}$ in (9) can be reduced to a single sum according to 


$$
\begin{aligned}
& \sum_{n_{1}, n_{2}=-\infty}^{\infty} J_{n_{1}}\left(\alpha_{l}\right) J_{n_{2}}\left(-\beta_{l}\right) \exp \left(i n_{1} \omega_{0} t+i n_{2}\left(\omega_{0} t+\pi / 2\right)\right)= \\
& \sum_{n=-\infty}^{\infty} J_{n}\left(w_{l}\right) \exp \left(i n\left(\omega_{0} t-\xi_{l}\right)\right),
\end{aligned}
$$

where

$$
\begin{aligned}
w_{l} & =\sqrt{\alpha_{l}^{2}+\beta_{l}^{2}} \\
\tan \xi_{l} & =\frac{\beta_{l}}{\alpha_{l}}=\mathbf{p}_{l} \cdot \mathbf{r} \\
\mathbf{p}_{l} & =\frac{\mathbf{F}_{0}}{\alpha_{l} \omega_{0}} .
\end{aligned}
$$

With the help of the identity (10) one can perform the integration over time in (4) and the $S$-matrix element is transformed into

$$
\begin{aligned}
S_{f i}= & \frac{i Z_{p}}{\pi V} \sum_{n, n_{3}} J_{n_{3}}\left(\gamma_{l}\right) \frac{1}{\mathbf{q}^{2}} \int d^{3} \mathbf{r} \varphi_{\mathbf{k}}^{*}(\mathbf{r}) J_{n}\left(w_{l}\right) \exp \left(i\left(\mathbf{q} \cdot \mathbf{r}-n \xi_{l}\right)\right) \varphi_{0}(\mathbf{r}) \\
& \delta\left(E_{f}+\varepsilon_{k}-E_{i}-\varepsilon_{0}+n \omega_{0}+2 n_{3} \omega_{0}\right) .
\end{aligned}
$$

Expression (12) can be substantially simplified if we note the following. We have already assumed that, on the target scale, the electron velocity in the final state $\sim k$ is very high and the field frequency is very low. Therefore the absolute value of the parameter $\alpha_{l}$ will, in general, be much larger than that of $\beta_{l}$. Assuming that this is the case, $\left|\alpha_{l}\right| \gg\left|\beta_{l}\right|$, one has $w_{l} \approx|\alpha|$ and the Bessel functions $J_{n}$ in (12) become coordinate-independent. Further, if we assume that $\left|\alpha_{l}\right| \gg\left|\gamma_{l}\right|$, then, following the argumentation given in [6], one can neglect the $n_{3}$-dependence in the delta-function. Then the sum over $n_{3}$ in (12) reduces to the phasefactor $\exp \left(i \frac{F_{0}^{2}}{8 \omega_{0}^{3}} \sin \left(2 \varphi_{0}\right)\right)$ which is inessential for the calculation of the cross section and can be omitted. Thus, we have

$$
\begin{aligned}
S_{f i}= & \frac{i Z_{p}}{\pi V} \sum_{n} J_{n}\left(\left|\alpha_{l}\right|\right) \frac{1}{\mathbf{q}^{2}} \int d^{3} \mathbf{r} \varphi_{\mathbf{k}}^{*}(\mathbf{r}) \exp \left(i\left(\mathbf{q}-n \mathbf{p}_{l}\right) \cdot \mathbf{r}\right) \varphi_{0}(\mathbf{r}) \\
& \delta\left(E_{f}+\varepsilon_{k}-E_{i}-\varepsilon_{0}+n \omega_{0}\right) .
\end{aligned}
$$

Using the standard techniques in order to get cross section from a known $S$-matrix element and taking into account that for a heavy particle impact one has $E_{i}-E_{f}=\mathbf{v} \cdot \mathbf{q}$, we obtain

$$
\frac{d^{3} \sigma}{d \mathbf{k}}=\frac{4 Z_{p}^{2}}{v^{2}} \sum_{n=-\infty}^{+\infty} J_{n}^{2}\left(\alpha_{l}\right) \int d^{2} \mathbf{Q} \frac{\left|\left\langle\varphi_{\mathbf{k}}\left|\exp \left(i\left(\mathbf{q}-n \mathbf{p}_{l}\right) \cdot \mathbf{r}\right)\right| \varphi_{0}\right\rangle\right|^{2}}{q^{4}}
$$

where

$$
\mathbf{q}=\mathbf{q}_{n}=\left(\mathbf{Q}, \frac{\varepsilon_{k}-\varepsilon_{0}+n \omega_{0}}{v}\right)
$$

and $\mathbf{Q}$ is the two-dimensional transverse part of the momentum transfer, $\mathbf{Q} \cdot \mathbf{v}=0$. Using a different approach the formula (14) was previously obtained in [6]. 


\section{B. Circular polarization}

In case of circular polarization we take the vector potential as $\mathbf{A}(t)=\mathbf{A}_{01} \cos \omega_{0} t+$ $\mathbf{A}_{02} \cos \left(\omega_{0} t+\pi / 2\right)$, where $\mathbf{A}_{0 j}=\frac{c \mathbf{F}_{0 j}}{\omega_{0}}, \mathbf{F}_{0 j}(j=1,2)$ are the amplitudes of the field oscillations in two mutually perpendicular directions, $\mathbf{F}_{01} \cdot \mathbf{F}_{02}=0$, and $F_{01}=F_{02}=F_{0}$. Similarly to the previous subsection we have

$$
\begin{aligned}
& \exp \left(-\frac{i}{c} \mathbf{A}(t) \cdot \mathbf{r}\right) \exp \left(i \frac{\mathbf{k}}{c} \int^{t} d t^{\prime} \mathbf{A}\left(t^{\prime}\right)\right) \exp \left(\frac{i}{2 c^{2}} \int^{t} d t^{\prime} A^{2}\left(t^{\prime}\right)\right) \\
& =\exp \left(i \frac{F_{0}^{2}}{2 \omega_{0}^{2}} t\right) \sum_{n_{1}, n_{2}=-\infty}^{\infty} J_{n_{1}}\left(\alpha_{c}\right) J_{n_{2}}\left(\beta_{c}\right) \exp \left(i n_{1}\left(\omega_{0} t+\chi_{1}\right)+i n_{2}\left(\omega_{0} t-\chi_{2}\right)\right)
\end{aligned}
$$

In Eq.(16) it is denoted

$$
\begin{aligned}
\alpha_{c} & =\sqrt{\left(\frac{\mathbf{F}_{01} \cdot \mathbf{k}}{\omega_{0}^{2}}\right)^{2}+\left(\frac{\mathbf{F}_{02} \cdot \mathbf{k}}{\omega_{0}^{2}}\right)^{2}} \\
\beta_{c} & =\sqrt{\left(\frac{\mathbf{F}_{01} \cdot \mathbf{r}}{\omega_{0}}\right)^{2}+\left(\frac{\mathbf{F}_{02} \cdot \mathbf{r}}{\omega_{0}}\right)^{2}} \\
\tan \chi_{1} & =\frac{\mathbf{F}_{02} \cdot \mathbf{k}}{\mathbf{F}_{01} \cdot \mathbf{k}} \\
\tan \chi_{2} & =\frac{\mathbf{F}_{01} \cdot \mathbf{k}}{\mathbf{F}_{02} \cdot \mathbf{r}} .
\end{aligned}
$$

The double sum in (16) can be reduced to a single sum, as before, with the help of Graf's theorem. The result is

$$
\begin{aligned}
& \sum_{n_{1}, n_{2}=-\infty}^{\infty} J_{n_{1}}\left(\alpha_{c}\right) J_{n_{2}}\left(-\beta_{c}\right) \exp \left(i n_{1}\left(\omega_{0} t+\chi_{1}\right)+i n_{2}\left(\omega_{0} t-\chi_{2}\right)\right) \\
= & \sum_{n=-\infty}^{\infty} J_{n}\left(w_{c}\right) \exp \left(i n\left(\omega_{0} t-\xi_{c}\right)\right) .
\end{aligned}
$$

Here

$$
\begin{aligned}
w_{c} & =\sqrt{\alpha_{c}^{2}+\beta_{c}^{2}+2 \alpha_{c} \beta_{c} \cos \left(\chi_{1}+\chi_{2}\right)} \\
\tan \xi_{c} & =\frac{\beta_{c} \sin \left(\chi_{1}+\chi_{2}\right)}{\alpha_{c}+\beta_{c} \cos \left(\chi_{1}+\chi_{2}\right)} .
\end{aligned}
$$

Provided the condition $\alpha_{c} \gg \beta_{c}$ is fulfilled, the parameter $w_{c}$ can be replaced by $\alpha_{c}$ and the phase-factor $\xi_{c}$ also takes on the comparatively simple form:

$$
\begin{aligned}
& w_{c}=\alpha_{c} \\
& \xi_{c}=\mathbf{p}_{c} \cdot \mathbf{r}=\frac{1}{\alpha_{c}^{2} \omega_{0}^{3}}\left(\left(\mathbf{F}_{01} \cdot \mathbf{k}\right) \mathbf{F}_{01}+\left(\mathbf{F}_{02} \cdot \mathbf{k}\right) \mathbf{F}_{02}\right) \cdot \mathbf{r}
\end{aligned}
$$

Now the transition $S$-matrix element and the cross section are given by 


$$
\begin{aligned}
S_{f i}= & \frac{i Z_{p}}{\pi V} \sum_{n} J_{n}\left(\alpha_{c}\right) \frac{1}{\mathbf{q}^{2}} \int d^{3} \mathbf{r} \varphi_{\mathbf{k}}^{*}(\mathbf{r}) \exp \left(i\left(\mathbf{q}-n \mathbf{p}_{c}\right) \cdot \mathbf{r}\right) \varphi_{0}(\mathbf{r}) \\
& \delta\left(E_{f}+\varepsilon_{k}-E_{i}-\varepsilon_{0}+n \omega_{0}+\frac{F_{0}^{2}}{2 \omega_{0}^{2}}\right)
\end{aligned}
$$

and

$$
\frac{d^{3} \sigma}{d \mathbf{k}}=\frac{4 Z_{p}^{2}}{v^{2}} \sum_{n=-\infty}^{+\infty} J_{n}^{2}\left(\alpha_{c}\right) \int d^{2} \mathbf{Q} \frac{\left|\left\langle\varphi_{\mathbf{k}}\left|\exp \left(i\left(\mathbf{q}_{n}-n \mathbf{p}_{c}\right) \cdot \mathbf{r}\right)\right| \varphi_{0}\right\rangle\right|^{2}}{q^{4}}
$$

respectively. In Eq.(22)

$$
\mathbf{q}_{n}=\left(\mathbf{Q}, \frac{\varepsilon_{k}-\varepsilon_{0}+n \omega_{0}+\frac{F_{0}^{2}}{2 \omega_{0}^{2}}}{v}\right)
$$

where $\mathbf{Q}$ is the transverse part of the momentum transfer, $\mathbf{Q} \cdot \mathbf{v}=0$.

For the case of circular polarization the treatment of the interaction term $A^{2} / 2 c^{2}$ represents no difficulty resulting just in the additional term $\frac{F_{0}^{2}}{2 \omega_{0}^{2} v_{p}}$ in the longitudinal component of the momentum transfer $\mathbf{q}$. This gives the possibility to easily study the importance of the term $A^{2}(t) / 2 c^{2}$ for the case of circular polarization by comparing results obtained with and without the correction $F_{0}^{2} /\left(2 \omega_{0}^{2} v_{p}\right)$. Such a study can also bring some ideas about the importance of the interaction term $A^{2}(t) / 2 c^{2}$ for the cases of linearly and elliptically polarized fields.

\section{Elliptic polarization}

In case of elliptic polarization $\mathbf{A}(t)=\frac{c \mathbf{F}_{01}}{\omega_{0}} \cos \omega_{0} t+\frac{c \mathbf{F}_{02}}{\omega_{0}} \cos \left(\omega_{0} t+\pi / 2\right)$, where $\mathbf{F}_{01} \cdot \mathbf{F}_{02}=0$ and, in general, $F_{01} \neq F_{02}$. To obtain the cross section for the collisions assisted by an elliptically polarized field one can use the results already derived for the case of circular polarization if we note the following. Although the electromagnetic field was described in the previous subsection as circularly polarized, the condition $F_{01}=F_{02}$ was, in fact, used only for considering the $\mathbf{A}^{2}(t)$ term. Therefore, if we assume, as in the treatment of a linearly polarized field, that the term $\mathbf{A}^{2}(t)$ can be neglected, the electron cross section for the case of elliptic polarization is given by

$$
\frac{d^{3} \sigma}{d \mathbf{k}}=\frac{4 Z_{p}^{2}}{v^{2}} \sum_{n=-\infty}^{+\infty} J_{n}^{2}\left(\alpha_{e l}\right) \int d^{2} \mathbf{Q} \frac{\left|\left\langle\varphi_{\mathbf{k}}\left|\exp \left(i\left(\mathbf{q}_{n}-n \mathbf{p}_{e l}\right) \cdot \mathbf{r}\right)\right| \varphi_{0}\right\rangle\right|^{2}}{q^{4}}
$$

In Eq.(24)

$$
\alpha_{e l}=\sqrt{\left(\frac{\mathbf{F}_{01} \cdot \mathbf{k}}{\omega_{0}^{2}}\right)^{2}+\left(\frac{\mathbf{F}_{02} \cdot \mathbf{k}}{\omega_{0}^{2}}\right)^{2}}
$$

and 


$$
\begin{aligned}
\mathbf{q}_{n} & =\left(\mathbf{Q}, \frac{\varepsilon_{k}-\varepsilon_{0}+n \omega_{0}}{v}\right) \\
\mathbf{p}_{e l} & =\frac{1}{\alpha_{e l}^{2} \omega_{0}^{3}}\left(\left(\mathbf{F}_{01} \cdot \mathbf{k}\right) \mathbf{F}_{01}+\left(\mathbf{F}_{02} \cdot \mathbf{k}\right) \mathbf{F}_{02}\right) .
\end{aligned}
$$

If $F_{01}=F_{02}=0$, the cross section (24) reduces to that for the field-free collisions. If $\mathbf{F}_{02}=0$ (or $\mathbf{F}_{01}=0$ ) the formulas (24)-(26) describe the electron emission in collisions assisted by a linearly polarized field.

\section{RESULTS AND DISCUSSION}

The cross sections (14), (22) and (24) were derived by using the velocity gauge. In the length gauge the initial electron state would be given by [6]

$$
\psi_{i}^{L}(t)=\varphi_{0}(\mathbf{r}) \exp \left(-i \varepsilon_{0} t\right)
$$

The factor $\exp \left(-i \frac{1}{c} \mathbf{A}(t) \cdot \mathbf{r}\right)$ is now missing in the initial electron wavefunction. However, in the length gauge the factor $\exp \left(i \frac{1}{c} \mathbf{A}(t) \cdot \mathbf{r}\right)$ appears in the Volkov solution for a free electron in the field of a plane electromagnetic wave. Correspondingly, the final state, taken in the Coulomb-Volkov approximation, in the length gauge reads

$\psi_{f}^{L}(t)=\varphi_{\mathbf{k}}(\mathbf{r}) \exp \left(-i \varepsilon_{k} t\right) \exp \left(i \frac{1}{c} \mathbf{A}(t) \cdot \mathbf{r}\right) \exp \left(-i \frac{\mathbf{k}}{c} \cdot \int_{+\infty}^{t} d t^{\prime} \mathbf{A}\left(t^{\prime}\right)-\frac{i}{2 c^{2}} \int_{+\infty}^{t} d t^{\prime} A^{2}\left(t^{\prime}\right)\right)$.

It is not difficult to see that, within the approach used in this paper, calculations with the states (27) and (28) would result in the same transition amplitudes and cross sections as those with the states (7) and (8). Thus, in our case both the velocity and lenght gauges lead to equivalent results.

In the derivation of the cross section for the cases of linear and elliptic polarizations we assumed that the influence of the term $A^{2}(t) / 2$ can be neglected, i.e. that $|\alpha| \gg\left(F_{01}^{2}+F_{02}^{2}\right) /\left(8 \omega_{0}^{3}\right)$ where $\alpha=\alpha_{l}, \alpha_{e l}$. Hence the applicability of expressions (14) and (24) is restricted to not too low frequencies (e.g. for the case of linear polarization to $\left.\omega_{0} \gg \frac{1}{8} \frac{F_{0}^{2}}{\mathbf{F}_{0} \cdot \mathbf{k}}\right)$. There is a more general restriction on the frequency which is applied also for the case of circular polarization. This limitation is not easily seen in the present treatment where the projectile motion is described quantum mechanically. However, it directly follows from the semi-classical treatment of [6] where it was found that the condition $\omega_{0} \gg \Omega=2 \pi v_{p} / d$ ( $d$ is the size of the laser focal spot) is necessary to obtain the cross section (14). This, in particular, means that Eqs.(14), (22) and (24) cannot be used to consider the case of a constant electric field $\omega_{0}=0$. In principle, for collisions assisted by a laser field with circular polarization one could use the cross section (22) to consider the limit of very low frequencies (but still not the point $\omega_{0}=0$ ). Although such an electromagnetic field would not be completely equivalent to a constant field, some properties of the emission, predicted by Eq.(22), are already rather close to those in the case of a constant field. For example, as will be discussed in detail below, the cross section (22) (and also (14) and (24)) predicts that the energy exchange with the electromagnetic field in the collision is inversely 
proportional to the field frequency. This can be compared to the infinite energy exchange between a free electron and a constant, unrestricted in space, electric field.

The electromagnetic field was described as a classical field. However, the amount of energy $n \omega_{0}$, which is exchanged between the colliding system and the electromagnetic field turns out to be quantized and we may speak about the number $|n|$ of photons emitted or absorbed during the electron emission. The parameter $\alpha\left(\alpha_{l}, \alpha_{c}\right.$ and $\left.\alpha_{e l}\right)$ describes the effective strength of the coupling between the colliding system and the electromagnetic field. According to the properties of the Bessel functions $J_{n}$, a maximum number of photons which can be exchanged between the colliding system and the field is of the order of $|\alpha|$.

We mentioned already that in the rest frame of the projectile the process in question can be viewed as 'bound-free' electron-ion scattering. It is of interest to compare this process with the free-free electron-ion scattering. The cross section for the latter process is given by ( see e.g. [5] and references therein):

$$
d \sigma=\sum_{n} \frac{p^{\prime}}{p} J_{n}^{2}(\beta) d \sigma_{e l} .
$$

In this expression the parameter $\beta$ (which for the case of linearly polarized field takes on the very simple form $\beta=\frac{\left|\mathbf{F}_{0} \cdot \Delta \mathbf{p}\right|}{\omega_{0}^{2}}$ ) describes the effective strength of the coupling between the electron and electromagnetic field in the scattering process. Further, $\mathbf{p}$ and $\mathbf{p}^{\prime}$ are the initial and final electron momenta, $\Delta \mathbf{p}=\mathbf{p}^{\prime}-\mathbf{p}, \mathbf{F}_{0}$ and $\omega_{0}$ are the amplitude and frequency of the field, $J_{n}$ are the Bessel functions, and $d \sigma_{e l}$ is the differential cross section of the electron-ion scattering in the absence of the field. The summation in (29) is to be performed over the number $n$ of photons exchanged between the electron and the electromagnetic field.

It is seen that the structure of the cross sections for free-free and 'bound-free' scattering has a certain similarity. In both cases the cross section is given by the sum over processes with the exchange of different numbers of photons. The form of the coupling parameters $\alpha$ and $\beta$ is also quite similar, e.g. for a linearly polarized field $\left|\alpha_{l}\right|=\frac{\left|\mathbf{F}_{0} \cdot \mathbf{k}\right|}{\omega_{0}^{2}} \approx \frac{\left|\mathbf{F}_{0} \cdot \Delta \mathbf{k}\right|}{\omega_{0}^{2}}$ and $\beta=\frac{\left|\mathbf{F}_{0} \cdot \Delta \mathbf{p}\right|}{\omega_{0}^{2}}$, where $\Delta \mathbf{k}$ and $\Delta \mathbf{p}$ are the changes in the electron momentum. At the same time the free-free electron scattering and the process under consideration have very important differences. That, in particular, is reflected in the theoretical description. Here we mention two points. First, the terms $\exp (-i n \mathbf{p} \cdot \mathbf{r})$ in Eqs.(14), (22) and (24), whose appearance can be traced back to originate in the factor $\exp \left(-i \frac{1}{c} \mathbf{A}(t) \mathbf{r}\right)$ in $(7)$, will be proven below to have a crucial effect on the description of the 'bound-free' scattering already at $F_{0} \sim \omega_{0}$. In the consideration of the free-free scattering, however, such terms do not appear at all. Second, in the treatment of the field-assisted free-free electron scattering in the dipole approximation, the interaction term $\frac{A^{2}(t)}{2 c^{2}}$ has no influence on the scattering cross section (see e.g. [5]) since it results just in the time-independent phase-factor $\exp \left(-\left(i / 2 c^{2}\right) \int_{-\infty}^{+\infty} d t A^{2}(t)\right)$ which finally drops out in the calculation. In contrast, in the treatment of the field-assisted 'bound-free' transitions this interaction term cannot be simultaneously removed from both the initial and final electron wavefunctions. That may bring substantial complications since this interaction term should, in general, be taken into account and can considerably affect the electron emission cross section at $F_{0} / \omega_{0} \gg 1$. Clearly, these formal differences in the description of the free-free and 'bound-free' scattering processes are just a reflection of the difference between a free and a bound electron and of their different response to the field of a weak 
low-frequency electromagnetic wave.

Let us now go over to discuss some examples of spectra for electrons emitted in fieldassisted collisions.

\section{A. Collisions assisted by a linearly polarized field}

In the field-free electron emission the triply-differential cross section reduces, in fact, to the doubly-differential cross section since the electron spectrum depends only on the emission energy and the angle $\vartheta_{\mathbf{k}}$ between the electron and projectile velocities. In the field-assisted collisions the collision geometry becomes more complicated and, in addition to the 'polar' angle $\vartheta_{\mathbf{k}}$, a nontrivial dependence of the electron spectrum on the 'azimuthal' emission angle can also be expected. To begin with, however, we restrict our attention to the field-assisted emission in collisions where the field vector $\mathbf{F}_{0}$ is parallel to the projectile velocity $\mathbf{v}_{p}, \vartheta_{\mathbf{F}}=\arccos \left(\frac{\mathbf{F}_{0} \cdot \mathbf{v}_{p}}{F_{0} v_{p}}\right)=0^{0}$, and where the electron spectrum is a function of the emission energy $\varepsilon_{k}$ and the angle $\vartheta_{\mathbf{k}}$.

In figures 1a-c the energy spectra of electrons, emitted in $\mathrm{He}^{2+}+\mathrm{H}(1 \mathrm{~s}) \rightarrow \mathrm{He}^{2+}+\mathrm{p}+$ $\mathrm{e}^{-}$collisions, are shown for a collision velocity $v_{p}=12$ a.u.. The frequency of the field is taken to be that of a $\mathrm{CO}_{2}$ laser, $\omega_{0}=0.004$ a.u. $\left(\omega_{0} \approx 0.11 \mathrm{eV}\right)$. The field strength is equal to $0,0.005$ and 0.008 a.u.. For emission angles close to zero the set of the vectors $\mathbf{F}_{0}, \mathbf{v}_{p}$ and $\mathbf{k}$ provides the largest values of the coupling parameter $\left|\alpha_{l}\right|$ which are possible for fixed $F_{0}, \omega_{0}$ and $v_{p}$. Therefore, we first consider the emission at zero emission angle $\vartheta_{\mathbf{k}}=0^{0}$. The cross sections were calculated numerically using Eq.(14) and the corresponding cross section for the binary-encounter emission in the field-free collisions.

As seen in figure 1a, the energy spectrum can be substantially modified by the presence of the field. For the collision geometry under consideration, as it was already pointed out in [6], a strong modification occurs if the parameter $\Delta E=\left|\alpha_{l}\right| \omega_{0}$ characterizing the energy exchange between the colliding system and the field, is not considerably smaller than the width of the electron spectrum, $\delta E \sim k v_{0}$, in the field-free collisions. This width is due to the target Compton profile and for ionization from the hydrogen ground state one has $v_{0} \sim 1$. For $\Delta E>\delta E$ the double-peak structure replaces the usual single peak of the field-free electron emission. Due to the interaction with the field the electron energy spectrum is now split with appearance of two maxima which are centered at $E_{1,2} \approx 2 v_{p}^{2} \pm \Delta E$. The formal origin of the double-peak structure in the calculation can be traced to appear due to the property of the Bessel function $J_{n}(x)$ at large $|n|^{3}$. One can also note that, due to this property, the quantity $\Delta E=\left|\alpha_{l}\right| \omega_{0}$ can be thought of as representing a typical energy exchange with the field ${ }^{4}$. In the case under consideration $\Delta E \sim 0.6$ and $1.1 \mathrm{keV}$ for $F_{0}=0.005$ and 0.008

\footnotetext{
${ }^{3}$ Numerical calculations show that the function $J_{n}^{2}(x)$ reaches its maximum values at $|x|$ which are less but close to $|n|$.

${ }^{4}$ By considering the interaction of the laser field with an electron which at time $t=t_{0}$ 'suddenly' changes its momentum (velocity) by $\Delta \mathbf{p}=\mathbf{k}$, where $|\mathbf{k}| \gg \frac{F_{0}}{\omega_{0}}$, the quantity $\Delta E$ can be directly related to the classical work performed by the field on the electron.
} 
a.u., respectively, and, indeed, these values are very relevant to the changes observed in the electron energy distributions displayed in figure 1a. The electromagnetic field becomes a very important 'third body' in the electron emission process. Thus, the binary-encounter character of the emission ceases to exist in the presence of a weak electromagnetic field.

In the collision-free regime the time of life $\tau$ of the ground state of hydrogen with respect to photoionization can be roughly estimated as $\tau \sim 0.25 \sqrt{F_{0}} \exp \left(2 /\left(3 F_{0}\right)\right)$ in the tunneling limit and as $\tau \sim\left(1 / F_{0}\right)^{N}$, where $N \sim 0.5 / 0.004 \sim 100$, in the multiphoton limit (see e.g. [10]). Assuming that the tunneling limit fits better for $F_{0}=0.008$ a.u. and $\omega_{0}=0.004$ a.u., we obtain $\tau \sim 5.1 \times 10^{39}$ a.u. $\sim 10^{19}$ s. Thus, an electromagnetic field with parameters taken in our examples would have practically no effect on the collision-free target. The results for the emission suggest, however, that such a field can be very strongly coupled to the colliding projectile-target system. This strong coupling, leading to the exchange of a large amount of energy between the field and the electron, profoundly modifies the electron emission spectra.

In figure $1 \mathrm{~b}$ the energy spectrum is shown for the same collision system and field parameters but for an emission angle $\vartheta_{\mathbf{k}}=30^{\circ}$. In this case the effect of the field on the electron spectra is substantially weaker compared to that at $\vartheta_{\mathbf{k}}=0^{0}$. This weakening is connected with two main reasons. One of the reasons is that for $\vartheta_{\mathbf{k}}=30^{0}$ the coupling parameter $\left|\alpha_{l}\right|$ is considerably smaller compared to that at $\vartheta_{\mathbf{k}}=0^{0}$. The second reason is that the angle $\vartheta_{\mathbf{k}}=30^{0}$ is not very far from an angle $\vartheta_{\mathbf{k}}=45^{\circ}$. The field-assisted emission at the latter angle has an interesting property. In the collision geometry, where the field polarization is parallel to the collision velocity, for the electron emission angles very close to $45^{0}$, there occurs a near cancellation in the exponentials $\exp \left(i\left(\mathbf{q}_{n}-n \mathbf{p}_{l}\right) \cdot \mathbf{r}\right)$ in Eq.(14) between $n \mathbf{p}_{l}$ and the $n$-dependent part of the longitudinal component of the momentum transfer $\mathbf{q}_{n}$. As a result, the numerator of the integrand in (14) becomes to be very weakly dependent on $n$. In addition, since in our treatment we assume that $F_{0} / \omega_{0} \ll k$, the energy exchange with the field is much smaller than the total change in the electron energy, $\Delta E \ll \varepsilon_{k}-\varepsilon_{0}$, and the denominator is practically $n$-independent as well. This leads to the very weak dependence of the integrand in (14) on the number of exchanged photons $n$. As a result, the energy spectra of electrons, emitted at angles very close to $\vartheta_{\mathbf{k}}=45^{0}$ are only very weakly changed compared to those in the field-free collisions (see figure 1c). At the same time the typical energy exchange with the field can still be very large (e.g. for $F_{0}=0.008$ a.u., $\omega_{0}=0.004$ a.u. and $\vartheta_{\mathbf{k}} \sim 45^{0}$ one has $\Delta E \sim 0.5 \mathrm{keV}$ ). One of possible explanations of the situation, where the substantial energy exchange with the field does not result in considerable modifications of the characteristics of the emitted electrons, would be that the emission at these angles represents the collision process, where the field exchanges the energy mainly with the projectile. Such a situation would be rather unexpected and interesting since the direct interaction between the heavy projectile and the electromagnetic field is comparatively very weak (and it has been neglected at all in our treatment) and the electron is the only particle which is directly coupled to the field. The realization of such a situation would mean that the heavy projectile can be very effectively coupled indirectly to the field using the electron as a mediator.

By inspecting Figures 1a-c one can see that, despite the profound effect on the energy distribution, the total number of the high-energy electrons emitted at a certain angle is practically not changed. This observation might mean that the field can have only a weak impact on the total number of the 'binary'-encounter electrons. 
In figures 2a-c we display the angular distributions of electrons with a fixed emission energy $\varepsilon_{k}=6700,7800$ and $9000 \mathrm{eV}$. The collision system and field are the same as in Figure 1. These figures show that the angular distribution can also be strongly affected by a weak low-frequency field. The changes in the angular spectra reflect the modification of the electron energy spectrum.

Let us now say a few words about the influence of the factor $\exp \left(-\frac{i}{c} \mathbf{A}(t) \cdot \mathbf{r}\right)$ (i.e. of the terms $\left.\exp \left(-i n \mathbf{p}_{l} \cdot \mathbf{r}\right)\right)$ on the cross section. This point was already briefly touched on when we discussed the peculiarities of the emission at angles close to $\vartheta_{\mathbf{k}}=45^{0}$, and it is of special interest because the presence of this factor is inherent to the 'bound-free' electron scattering. In figures 1a-c we compare results of our calculations for $F_{0}=0.008$ a.u. with and without keeping the term $\exp \left(-i \frac{1}{c} \mathbf{A}(t) \mathbf{r}\right)$ (dotted and dot-dashed curves, respectively). It is seen in the figures that, already at $F_{0} / \omega_{0} \sim 1$, the neglect of this term can completely change the shape of the electron energy distribution. Since it is assumed that $\omega_{0} \ll \omega_{\text {at }}$, where $\omega_{a t} \sim 1$ a.u. is the typical atomic transition frequency, this term cannot be neglected even for rather weak fields where $F_{0} \sim \omega_{0}$ and only for $F_{0} \ll \omega_{0}$ its influence vanishes. The neglect of the term $\exp \left(-i \frac{1}{c} \mathbf{A}(t) \mathbf{r}\right)$ in $(7)$ would correspond to taking the initial state in the length gauge while the final state $(8)$ is still taken in the velocity gauge. ${ }^{5}$ Thus, the necessity to keep this term can be formulated as the need to give a gauge-consistent treatment.

In figure 3 the electron energy spectra are shown for the same collision system, $\mathrm{He}^{2+}+$ $\mathrm{H}(1 \mathrm{~s}) \rightarrow \mathrm{He}^{2+}+\mathrm{p}+\mathrm{e}^{-}$, and a collision velocity, $v_{p}=12$ a.u.. Now, however, the angle between the collision velocity and the field polarization is nonzero, $\vartheta_{\mathbf{F}}=30^{\circ}$, and the electron emission spectrum depends not only on the angle $\vartheta_{\mathbf{k}}$ between the electron momentum $\mathbf{k}$ and the projectile velocity $\mathbf{v}_{p}$ but also on the angle $\varphi_{k}$ between $\mathbf{k}$ and the plane formed by the vectors $\mathbf{v}_{p}$ and $\mathbf{F}_{0}$. Comparing electron energy distributions for emission at $\vartheta_{\mathbf{k}}=30^{0}$ for the cases $\vartheta_{\mathbf{F}}=0^{0}$ and $\vartheta_{\mathbf{F}}=30^{0}$, we observe for the latter case a considerable increase in the modulation. This can be partly attributed to the stronger coupling with the field in the case $\vartheta_{\mathbf{F}}=30^{\circ}$, where $\mathbf{k} \| \mathbf{F}_{0}$. The other important reason is that in the collision geometry under consideration, there are no 'special' emission angles similar to that of $\vartheta_{\mathbf{k}}=45^{0}$ in the geometry where $\vartheta_{\mathbf{F}}=0^{0}$. Since the coupling parameter decreases with increasing the angle $\varphi_{k}$, we see in figure 3 that for larger emission angles $\varphi_{k}$ the electron spectrum is weaker affected by the field.

\section{B. Collisions assisted by a circularly polarized field}

To illustrate modifications of the electron emission in the presence of a circularly polarized electromagnetic field, we consider energy and angular distributions of electrons emitted in $\mathrm{He}^{2+}+\mathrm{H}(1 \mathrm{~s}) \rightarrow \mathrm{He}^{2+}+\mathrm{p}+\mathrm{e}^{-}$collisions, for a collision velocity $v_{p}=12$ a.u.. We choose a collision geometry, where one of the field components is parallel and the second is perpendicular to the collision velocity, and consider spectra of electrons emitted at different directions characterized by the angles $\vartheta_{\mathbf{k}}$ and $\varphi_{\mathbf{k}}$, where the latter is the angle between the

\footnotetext{
${ }^{5}$ According to Eqs.(27) and (28) the neglect of this term can be also thought of as taking the initial state in the velocity gauge and the final state in the length gauge.
} 
final electron momentum and the plane formed by the collision velocity and the field vectors. The field frequency and strength are: $\omega_{0}=0.004 \mathrm{a} . \mathrm{u}$. and $F_{0}=0,0.005$ and 0.008 a.u. The cross sections were calculated numerically using Eq.(22).

For an emission angle $\vartheta_{\mathbf{k}}=0^{0}$, the electron energy spectra are shown in Figure 4a. As seen in this figure, the changes in the energy spectrum are quite similar to those in the case of linear polarization. In Figure $4 \mathrm{~b}$ the energy spectrum is shown for the same collision system and field parameters but for an emission angle $\vartheta_{\mathbf{k}}=30^{\circ}$ and for angles $\varphi_{k}=0^{0}, 30^{0}$ and $45^{\circ}$.

The case of circular polarization is especially suitable to get the idea not only about the role of the terms $\exp \left(-i \mathbf{p}_{n} \cdot \mathbf{r}\right)$ but also about that of $A^{2} / 2 c^{2}$. The presence of both the terms is inherent to the 'bound-free' scattering. We performed calculations neglecting the terms $\exp \left(-i \mathbf{p}_{n} \cdot \mathbf{r}\right)$ (figure $\left.4 \mathrm{a}\right)$ and the term $A^{2} / 2 c^{2}$ (figure $4 \mathrm{~b}$ ). It is seen that at $F_{0} / \omega_{0} \sim 1$ the terms $\exp \left(-i \mathbf{p}_{n} \cdot \mathbf{r}\right)$ play a crucial role also for the proper treatment of the collisions assisted by a circularly polarized field. At $F_{0} \sim \omega_{0}$ the term $A^{2} / 2 c^{2}$ is of relatively lesser significance. ${ }^{6}$

In Figure 5 we show an example of the angular distributions of the emitted electrons, as a function of the emission angle $\vartheta_{\mathbf{k}}$, for a fixed emission energy $E=7800 \mathrm{eV}$ and for the emission angle $\varphi_{\mathbf{k}}=0^{\circ}$ and $90^{\circ}$. The collision system and field are the same as in Figure 4. It is seen that there is a certain similarity between the angular dependencies shown in figures $2 \mathrm{~b}$ and 5 . One can also observe a noticeable dependence of the electron emission on the 'second' emission angle $\varphi_{\mathbf{k}}$.

\section{Field-assisted emission in collisions with neutral projectiles}

In ion-atom collision, where the ion initially carries an electron, the electron emission can also be due to the electron removal from the ion. In order to get some preliminary ideas about the field-assisted binary-encounter emission in collisions with a neutral atomic particle, we considered the emission in collisions with a particle whose interaction with the target electron is given by

$$
W_{s}=-Z_{p} \frac{\exp (-\kappa|\mathbf{R}-\mathbf{r}|)}{|\mathbf{R}-\mathbf{r}|}
$$

Here $r_{a} \sim \frac{1}{\kappa}$ is the effective radius of the interaction. The potential (30) can be used as a rough approximation to describe the collision with a neutral atom-projectile which remains in its initial internal state during the collision process. We performed calculations for the field-assisted 'binary-encounter' emission from hydrogen in collisions with a neutral projectile with $\kappa \sim 1$ a.u. and found practically no difference between the collisions with $\kappa \sim 1$ and those with $\kappa=0$ considered in the previous subsections. This lack of difference can be

\footnotetext{
${ }^{6}$ At this point we note that a different approach to field-assisted atom ionization by a charged projectile (with the emphasis on electron impact) was formulated in [9]. That approach, however, disregards both the gauge factor $\exp \left(-\frac{i}{c} \mathbf{A}(t) \cdot \mathbf{r}\right)$ and the term $A^{2}(t) / 2 c^{2}$ and, in fact, is restricted to fields with $F_{0} \ll \omega_{0}$ where the field modification of the emission in question is negligible.
} 
understood if one notes that the collisions, resulting in a very high momentum transfer to the target electron, occur at impact parameters much smaller than $\frac{1}{\kappa} \sim 1$ a.u.. Therefore in

the considered example the difference between the pure Coulomb, $-\frac{Z_{p}}{|\mathbf{R}-\mathbf{r}|}$, and the screened Coulomb interaction (30) plays essentially no role.

\section{On the possibility to test predicted effects experimentally}

In an experiment, the predicted effects can be tested by crossing projectile and laser beams with a target gas. For a collision experiment on the field-assisted emission it is desirable to have a laser pulse with a duration not shorter than $\sim 1 \mathrm{~ns}$. According to our consideration, the substantial modification of the electron emission can be possible if $F_{0} \gtrsim \omega_{0}$. It means that, in general, at fixed field intensities, the effects are favoured for smaller field frequencies. For example, in order to keep the typical energy exchange with a field on the level of $\sim 1 \mathrm{keV}$ for the collision system discussed in the subsection IIIa, one should have a field intensity of $\sim 2 \times 10^{12}$ and $\sim 2 \times 10^{14} \mathrm{~W} / \mathrm{cm}^{2}$ for a field frequency of 0.1 and $1 \mathrm{eV}$, respectively. The latter intensity is already quite high and such a field can strongly deplete the ground state of a target before the collision with a projectile can occur. Because of this point, the use of lasers with frequencies of $1 \mathrm{eV}$ and higher seems to be rather questionable. One cannot take a field with a too low frequency either, since there is an important restriction $\omega_{0} \gg 2 \pi v_{p} / d[6]$. Assuming that $v=10$ a.u. $\approx 2.2 \times 10^{9} \mathrm{~cm} / \mathrm{s}$ and $d \sim 1 \mathrm{~mm}$, one obtains $\omega_{0} \gg 10^{11} \mathrm{~Hz}$. Therefore, it seems that $\mathrm{CO}_{2}$ lasers with $\omega_{0} \sim 2 \times 10^{14}$ $\mathrm{Hz}$ represent 'the golden mean'. Their frequency is not high and not too low, there exist $\mathrm{CO}_{2}$ lasers which can produce intensities $\sim 10^{12}-10^{13} \mathrm{~W} / \mathrm{cm}^{2}$ during a pulse with duration of a few nanoseconds. According to our estimates such intensities at a frequency of $\sim 0.1 \mathrm{eV}$ are sufficient to observe the considerable modifications in the 'binary-encounter' emission from light targets, like $\mathrm{H}_{2}$ and He.

\section{CONCLUSION}

We have considered modifications in energy and angular distributions of the 'binaryencounter' electron emission in fast ion-atom collisions in the presence of a low-frequency weak electromagnetic field. This consideration shows that a field, which would have no effect on the free target atom, can profoundly influence both the energy and angular spectra of emitted electrons. The process of the field-assisted 'binary-encounter' emission represents an example of the field-matter interaction where the numbers of photons involved and the energy exchange with the field are much larger than it is usually observed in the (impactfree) photoionization even at much higher field intensities (see e.g. [10], [11] and references therein).

In the field-assisted collisions the binary-encounter character of the electron emission ceases to exist because the field becomes an extreme important 'third body' in this process. The presence of the field might also be expected to influence the role of the target nucleus in the 'binary-encounter' emission in such a way that all the three particles could participate in the momentum-energy exchange. The field, described in the dipole approximation, cannot, of course, influence the momentum balance but may represent an important source and sink 
of energy. If the energy exchange between the colliding system and the field reaches considerable values, where $\Delta E \gtrsim \delta E \sim k v_{0}$, it is, as a rule, reflected by substantial modifications in the electron emission spectra. One can, however, mention the situation discussed in the subsection IIIa, where the considerable energy exchange with the field has only very weak impact on the shape of the electron spectra.

In the projectile frame the electron emission can be viewed as 'bound-free' electronion scattering. The structure of the cross section for the 'bound-free' scattering has some similarity to that of the free-free transitions. The analogy is, however, not very general since the free-free and 'bound-free' electron scattering have very important differences. In the formal mathematical treatment of the process these differences are mainly represented by the different roles played by the factor $\exp \left(-i \frac{1}{c} \mathbf{A}(t) \mathbf{r}\right)$ and the interaction term $\frac{A^{2}(t)}{2 c^{2}}$. These formal differences in the description of the free-free and 'bound-free' scattering processes are just a reflection of the difference in the response of a free electron and a bound electron to the field of a weak low-frequency electromagnetic wave. Profound modifications in the electron spectra can be observed at $F_{0} \gtrsim \omega_{0}$. For $F_{0} \sim \omega_{0}$ the factor $\exp \left(-i \frac{1}{c} \mathbf{A}(t) \mathbf{r}\right)$ plays already a crucial role in the treatment of the field-assisted electron emission. At such field parameters the role of the interaction term $\frac{A^{2}(t)}{2 c^{2}}$ is still relatively modest.

Existing sources of coherent electromagnetic radiation allow, in principle, to verify experimentally the predicted modifications in the field-assisted electron emission

\section{ACKNOWLEDGEMENTS}

The authors acknowledge with thanks the support from the Deutsche Forschungsgemeinschaft (DFG), the Max-Planck-Gesellschaft and the Leibniz-Program of the DFG. 


\section{REFERENCES}

[1] Stolterfoht N., DuBois R.D. and Rivarola R.D., Electron Emission in Heavy Ion-Atom Collisions, Springer-Verlag, Berlin Heidelberg (1997)

[2] Bransden B.H. and McDowell M.R.C., 1992, Charge Exchange and the Theory of IonAtom Collisions, Clarendon Press. Oxford

[3] Eichler J. and Meyerhof W.E., 1995, Relativistic Atomic Collisions, Academic Press San Diego

[4] McGuire J.H., Electron Correlation Dynamics in Atomic Collisions, Cambridge University Press (1997)

[5] Ehlotsky F., Jaron A., Kaminski J.Z., Phys.Rep. 297 63-153 (1998)

[6] Voitkiv A.B. and Ullrich J., J.Phys. B 341673 (2001)

[7] Volkov D.M., Z.Phys. 94250 (1935)

[8] Abramowitz M. and Stegun I. Handbook of Mathematical Functions, Dover Publications, Inc., New York (1964)

[9] Cavaliere P., Ferrante G. and Leone C., J.Phys. B 134495 (1980)

[10] Delone N.B. and Krainov V.P., Multiphoton processes in Atoms, Springer-Verlag, Berlin Heidelberg New York (2000)

[11] Joachain C.J., Dörr M. and Kylstra N., Adv.At.Mol.Opt.Phys., 42225 (2000) 


\section{FIGURE CAPTIONS}

Figure 1. Energy spectrum of the electron emission in $\mathrm{He}^{2+}+\mathrm{H}(1 \mathrm{~s}) \rightarrow \mathrm{He}^{2+}+\mathrm{p}+$ $\mathrm{e}^{-}$collisions assisted by a linearly polarized field. Collision velocity $v_{p}=12$ a.u., the field is polarized along the collision velocity, $\vartheta_{\mathbf{F}}=0^{0}$. (a) Emission angle $\vartheta_{\mathbf{k}}=0^{0}$. Solid curve: field-free collisions; dashed curve: collisions in the presence of a field with $F_{0}=0.005$ a.u. and $\omega_{0}=0.004$ a.u.; dotted curve: same as for dashed curve but for $F_{0}=0.008$ a.u.; dotdash curve: same as for dot curve but neglecting the terms $\exp \left(-i n \mathbf{p}_{l} \cdot \mathbf{r}\right)$. (b) Same as in (a) but for $\vartheta_{\mathbf{k}}=30^{\circ}$. (c) Same as in (a) but for $\vartheta_{\mathbf{k}}=45^{0}$.

Figure 2. Angular distribution of the electron emission in $\mathrm{He}^{2+}+\mathrm{H}(1 \mathrm{~s}) \rightarrow \mathrm{He}^{2+}+\mathrm{p}$ $+\mathrm{e}^{-}$collisions assisted by a linearly polarized field. Collision velocity $v_{p}=12$ a.u. and $\vartheta_{\mathbf{F}}=0^{0}$. (a) Emission energy $E=6700 \mathrm{eV}$. Solid curve: field-free collisions; dashed curve: collisions in the presence of a field with $F_{0}=0.005$ a.u. and $\omega_{0}=0.004$ a.u.; dotted curve: same as for dashed curve but for $F_{0}=0.008$ a.u.. (b) Same as in (a) but for $E=7800 \mathrm{eV}$. (c) Same as in (a) but for $E=9000 \mathrm{eV}$.

Figure 3. Energy spectrum of the electron emission in $\mathrm{He}^{2+}+\mathrm{H}(1 \mathrm{~s}) \rightarrow \mathrm{He}^{2+}+\mathrm{p}+\mathrm{e}^{-}$ collisions assisted by a linearly polarized field. Collision velocity $v_{p}=12$ a.u., $\vartheta_{\mathbf{F}}=30^{0}$ and $\vartheta_{\mathbf{k}}=30^{\circ}$. Solid curve: field-free collisions; dashed curve: collisions in the presence of a field with $F_{0}=0.008$ a.u. and $\omega_{0}=0.004$ a.u., $\varphi_{\mathbf{k}}=0^{0}$; dotted curve: same as for dashed curve but for $\varphi_{\mathbf{k}}=20^{\circ}$; dot-dash curve: same as for dot curve but for $\varphi_{\mathbf{k}}=30^{\circ}$; dot-dot-dash curve: same as for dot curve but for $\varphi_{\mathbf{k}}=45^{0}$.

Figure 4. Energy spectrum of the electron emission in $\mathrm{He}^{2+}+\mathrm{H}(1 \mathrm{~s}) \rightarrow \mathrm{He}^{2+}+\mathrm{p}+\mathrm{e}^{-}$ collisions assisted by a circularly polarized field. Collision velocity $v_{p}=12$ a.u.. (a) Emission angles $\vartheta_{\mathbf{k}}=0^{0}$ and $\varphi_{\mathbf{k}}=0^{0}$. Solid curve: field-free collisions; dashed curve: collisions in the presence of a field with $F_{0}=0.005$ a.u. and $\omega_{0}=0.004$ a.u.; dotted curve: same as for dashed curve but for $F_{0}=0.008$ a.u.; dot-dash curve: same as for dot curve but neglecting the terms $\exp \left(-i n \mathbf{p}_{c} \cdot \mathbf{r}\right)$. (b) The same collision system, emission angle $\vartheta_{\mathbf{k}}=30^{\circ}$. Solid curve: field-free collisions; dashed curve: collisions in the presence of a field with $F_{0}=0.005$ a.u. and $\omega_{0}=0.004$ a.u., $\varphi_{\mathbf{k}}=0^{0}$; dot curves: $F_{0}=0.008$ a.u., $\omega_{0}=0.004$ a.u. and $\varphi_{\mathbf{k}}=0^{0}$, $30^{0}$ and $45^{\circ}$; dot-dash curve: same as for the dot curve with $\varphi_{\mathbf{k}}=0^{0}$ but neglecting the term $A^{2} / 2 c^{2}$.

Figure 5. Angular spectrum of the electron emission in $\mathrm{He}^{2+}+\mathrm{H}(1 \mathrm{~s}) \rightarrow \mathrm{He}^{2+}+\mathrm{p}+\mathrm{e}^{-}$ collisions assisted by a circularly polarized field. Collision velocity $v_{p}=12$ a.u.. Emission energy $E=7800 \mathrm{eV}$. Solid curve: field-free collisions; dashed curve: collisions in the presence of a field with $F_{0}=0.005$ a.u. and $\omega_{0}=0.004$ a.u., $\varphi_{\mathbf{k}}=0^{0}$; dot curves: $F_{0}=0.008$ a.u. and $\omega_{0}=0.004$ a.u., $\varphi_{\mathbf{k}}=0^{0}$ and $90^{\circ}$. 